\title{
Combination of Cumulative Area Pre- Processing and Partial Least Squares for Handling Intensely Overlapping Binary and Ternary Drug Systems
}

\author{
Yahya S Al-Degs ${ }^{1 *}$, Amjad H. El-Sheikh ${ }^{1}$, Eman A. Abu Saaleek ${ }^{1}$, Reema A. Omeir ${ }^{1}$, \\ Musab A. Al-Ghodran' \\ 1 Chemistry Department, The Hashemite University, P. 0. Box 150459 Zarqa 13115 Jordan
}

\begin{abstract}
The analytical performance of cumulative area pre-processing (CAP), a recently developed signal filtering method, along with multivariate calibration for quantification of spectrally overlying drugs was outlined. The drug combinations containing high level of paracetamol (PAR) in the presence of caffeine (CAF), chlorpheniramine maleate (CHL), pseudoephedrine hydrochloride (PSE), phenylephrine hydrochloride (PHE), and diphenhydramine hydrochloride (DPH). The tested formulations were: PAR-CAF-PHE, PAR-CAF-PHE, and PAR-DRH. Based on netanalyte signal calculations, the formulations exhibited intense overlapping 53-68\% for PAR-PSE-CHL, 55-95\% for PAR-CAF-PHE, and 44\% for PAR-DRH. For each system, PLS latent variables were estimated using cross-validation technique and more factors were needed for highly overlapping systems. PLS-CAP was found applicable for drugs quantification in all systems with excellent performance regardless the size of spectral overlapping and ratios of components in the formulation. For PAR-PSE-CHL (ratio 300:30:2 mg/tablet), the ingredients were quantified by CAP-PLS with satisfactorily recoveries (RSD, $n=3$ ) 89.9\% (3.1\%), 104.6\% (2.7\%), and $99.0 \%$ (1.5\%) for PAR, PSE, and CHL, respectively. Both PLS and CAP-PLS were demonstrated the same performance for binary system of modest overlapping and no component available in low concentration.
\end{abstract}

Keywords: Signal Pre-processing; Spectral overlapping; Binary and Ternary Drug Formulations; PLS calibration

*Corresponding Author: Dr. Yahya Al-Degs, e-mail: yahyaaldeqs@yahoo.com

Yahya S. Al-Degs ORCID Number: 0000-0002-9555-7594

Amjad H. El-Sheikh ORCID Number: 0000-0001-8321-3236

Eman A. Abu Saaleek ORCID Number: 0000-0003-3163-2919

Reema A. Omeir ORCID Number: 0000-0002-3508-5927

Musab A. Al-Ghodran ORCID Number: 0000-0002-4766-0730

(Received 29 December 2018, accepted 24 January 2019) 


\section{INTRODUCTION}

Commercial drug formulations often contain more than one activates pharmaceutical ingredients (APIs) that present in variable levels to achieve the best pharmacological performance ${ }^{1}$. The spectral overlapping between the active ingredients is often moderate but intense overlapping is also possible. In addition to spectral overlapping and nonlinearity in the system, drug production stages like crystallization, drying, solid dosage form, added excipients, and tableting at different conditions can affect the spectral behavior of APIs ${ }^{1,2}$. Accordingly, accurate analytical methods are always needed in this regard ${ }^{1}$. The development and formulation of the pharmaceuticals brought a revolution in human health. These pharmaceuticals would help their intent only if they are free from impurities and are administered in an appropriate quantity. Hence, there are many challenges and it can be reduced by effective use of excipients, which permits formulators to overcome these challenges. It becomes necessary to develop new analytical methods because sometimes the dosage form contains other substances which potentially interfere in the assay and, if not corrected, may impart a systematic error to the assay ${ }^{1}$. Multi-component formulations have gained a lot of attention nowadays due to greater patient acceptability, increased potency, multiple action, quick relief and fewer side effects $^{1,2}$. Market is flooded with combination of drugs in various dosage form. One of such combination is paracetamol (PAR) with other drugs including caffeine (CAF), diphenhydramine hydrochloride (DPH), chlorpheniramine maleate (CHL), pseudoephedrine hydrochloride (PSE), phenylephrine hydrochloride (PHE). For example, PAR and DPH are co-formulated in pharmaceutical product for temporary relief of pain when associated with sleeping difficulty ${ }^{2-7}$. The combination PAR-CHL-PSE has been recently introduced in the market to treat the symptoms of most flues ${ }^{5-7}$. The combination PAR-CAF-PHE was also formulated to relief from major cold and flu symptoms and applied at day time as it doesn't cause drowsiness.

Various chemical and instrumental methods were developed to make drugs serve their purpose at regular intervals which are involved in the estimation of drugs. These pharmaceuticals may develop impurities at various steps of their development, transportation and storage, which makes the pharmaceutical risky to be administered. Thus, they must be identified and quantified. Hence, analytical instrumentation and methods play an important role. Thus, the review highlights a variety of analytical, chromatographic and instrumental method developed such as; High Performance Liquid Chromatography (HPLC), High Performance Thin Layer Chromatography (HPTLC), and Gas Chroma- 
tography (GC) have wide application in assuring the quality and quantity of pharmaceutical products and these instrumental methods are simple, precise, rapid, and reproducible and have been applied in the analysis of pharmaceuticals for assessing the quality of the drugs ${ }^{8-10}$.

Recently, multivariate calibration methods have been intensively employed for analyzing pharmaceutical formulations and detection of released drugs in urine and plasma ${ }^{5-7}$. Particularly speaking, PAR and CAF have been quantified in drug formulations using first-order multivariate calibration methods with high accuracy and without implementing any chromatographic procedure ${ }^{6,7}$. Compared to liquid chromatography, assaying drug formulations by multivariate calibration required less solvent consumption and avoids using tedious chromatographic instruments ${ }^{2,3}$. First order multivariate calibration methods includes multilinear regression (MLR), principal component regression (PCR), partial least-squares (PLS-1), and many others ${ }^{11}$. In a recent study, both PAR and PHE were quantified in four-drug formulations using PLS1 calibration ${ }^{5,6}$. PHE was quantified in the presence of PAR in tablet form with excellent recovery ${ }^{7}$. In another study, novel application of PCR and PLS was developed for determination of PAR, CAF, and CHL in real formulations containing other medicines ${ }^{6,7}$. In the drug formulation, the recovery and RSD values were 99.91\% (1.69\%), 100.15\%(1.35\%) and 100.49\%(1.66\%) for PAR, CAF and CHL, respectively. In the second method PLS was applied to quantify four medicines in real formulation with high recoveries and RSD: $99.80 \%$ (1.67\%), 100.19\% (1.42\%) and $100.45 \%$ (1.86\%) for PAR, CAF and CHL, respectively'. There is no published literature dealing with simultaneous quantification of PAR and DPH in real formulations. Spectral overlapping between drugs was reported to have a negative influence on the performance of multivariate calibration methods $^{11}$. Form the earlier studies, it is concluded that multivariate calibration is applicable for medicines determination in different commercial formulations including tablet and syrups.

Usually, the pre-processing methods are divided into three categories: a) filtering and de-noising, generally associated with the improvement of the signalto-noise ratio. These methods are applied to the raw data before the construction of the multivariate model and reduce the influence of the random variance without altering that useful, b) spectral normalization and differentiation, such as the spectral derivation and the application of the Fourier transform. These procedures are also applied before the construction of the model, and c) selection of the variables and reduction of dimensionality of the data. These methods are applied by multivariate modeling and therefore involve both the 
matrix X (independent variables) and the matrix Y (dependent variables). The most used method is orthogonal signal correction. There are a number of excellent review articles providing guidance for application of the pre-processing techniques to the analytical signals ${ }^{12-14}$. In some pharmaceutical formulations one or more components are present in very low concentrations, so as to be hidden by the components more concentrated or by the instrumental noise. This increases the difficulty of building a mathematical model able to predict in a satisfactory manner all the species present in the mixture. In these cases, the data pre-treatment should provide to amplify the analytical information due to the components at lower concentration and at the same time to minimize the spectral noise that can interfere with their determination.

In the current work, the application of newly proposed cumulative area preprocessing (CAP) will be evaluated for prior to PLS calibration for quantification of binary and ternary drugs systems. CAP was developed to amplify the spectral signals. CAP-PLS will be tested on the quantitative analysis of one binary and ternary pharmaceutical formulation. The common difficulty in analyzing these matrices was the presence of some components in much lower amount than other components. The binary formulation consisted of paracetamol-diphenhydramine hydrochloride (ratio 20:1), paracetamol-caffeinephenylephrine hydrochloride (ratio 100:5:1 mg/tablet), and paracetamol-pseudoephedrine hydrochloride-chlorpheniramine maleate (ratio 150:15:1 mg/ tablet). The analytical performance of CAP-PLS and PLS for drugs quantification will critically discussed. More attention will be paid on the influence spectral overlapping among drugs on their quantification by CAP-PLS. Influence of $\mathrm{pH}$ and spectral range on spectral analysis will be also considered.

\section{METHODOLOGY}

\section{Drugs and marketed formulations}

The pharmaceuticals paracetamol (PAR), caffeine (CAF), diphenhydramine hydrochloride (DPH), phenylephrine hydrochloride (PHE), pseudoephedrine hydrochloride (PSE), and chlorpheniramine maleate (CHL) were kindly donated from Dar Al-Dawaa company (Dar Al-Dawaa, Naour, Amman, Jordan). Distilled water was used for preparation of standard solutions and dissolution of commercial tablets. The marketed drugs were Panadol cold and fluß (Active ingredients: PAR-PSE-CHL 500:30:2 mg/tablet; excipients Silicon dioxide, stearic acid, sodium benzoate, povidone, starch, maize starch, and talc, GlaxoSmithKline Dungarvan Ltd., Co. Waterford, Ireland), Panadol cold and flu Dayß (Active ingredients: PAR-CAF-PHE 500:25:5 mg/tablet; excipients pre-gelatinised starch, maize starch, povidone, potassium sorbate, talc, stearic 
acid, microcrystalline cellulose, sodium lauryl sulphate, sunset yellow, GlaxoSmithKline Dungarvan Ltd., Co. Waterford, Ireland), and Panadol night ${ }^{\circledR}$ (PAR-DPH 500:25 mg/tablet; excipients maize starch, pregelatinised starch, potassium sorbate, povidone, talc, and stearic acid, GlaxoSmithKline Dungarvan Ltd., Co. Waterford, Ireland) All other reagents were of the highest purity commercially available. Table 1 summaries some chemical parameters of the undertaken pharmaceuticals.

Table 1. Chemical properties of studied pharmaceuticals.

\begin{tabular}{|c|c|c|c|c|}
\hline $\begin{array}{c}\text { Name/short } \\
\text { name }\end{array}$ & Formula & Structural formula & Medical action & $\mathbf{p K}_{\mathrm{a}}$ \\
\hline $\begin{array}{l}\text { Paracetamol } \\
\text { PAR }\end{array}$ & $\mathrm{C}_{8} \mathrm{H}_{9} \mathrm{O}_{2}$ & & $\begin{array}{l}\text { Treat mild to } \\
\text { moderate pains }\end{array}$ & 9.5 \\
\hline $\begin{array}{l}\text { Pseudoephedrine } \\
\text { hydrochloride } \\
\text { PSE }\end{array}$ & $\mathrm{C}_{10} \mathrm{H}_{15} \mathrm{ON}$ & & $\begin{array}{l}\text { Shrink blood } \\
\text { vessels in the nasal } \\
\text { passage (prevent } \\
\text { stuffy nose) }\end{array}$ & 10.3 \\
\hline $\begin{array}{c}\text { Chlorpheniramine } \\
\text { Maleate } \\
\text { CHL }\end{array}$ & $\mathrm{C}_{15} \mathrm{H}_{19} \mathrm{~N}_{2} \mathrm{Cl}$ & & $\begin{array}{l}\text { Relieve symptoms } \\
\text { of allergy }\end{array}$ & 9.1 \\
\hline $\begin{array}{l}\text { Caffeine } \\
\text { CAF }\end{array}$ & $\mathrm{C}_{8} \mathrm{H}_{10} \mathrm{~N}_{4} \mathrm{O}_{2}$ & & $\begin{array}{l}\text { Acts as central } \\
\text { nervous system } \\
\text { stimulant }\end{array}$ & 14.0 \\
\hline $\begin{array}{c}\text { Phenylephrine } \\
\text { hydrochloride } \\
\text { PHE }\end{array}$ & $\mathrm{C}_{9} \mathrm{H}_{14} \mathrm{CINO}_{2}$ & & $\begin{array}{l}\text { Sympathomimetic } \\
\text { (descongestants) }\end{array}$ & 9.07 \\
\hline $\begin{array}{l}\text { Diphenhydramine } \\
\text { Hydrochloride } \\
\text { DPH }\end{array}$ & $\mathrm{C}_{17} \mathrm{H}_{22} \mathrm{CINO}$ & & $\begin{array}{l}\text { Blocking the effects } \\
\text { of histamine and } \\
\text { causes drowsiness. }\end{array}$ & 9.13 \\
\hline
\end{tabular}




\section{Standard solutions, calibration/validation mixtures}

Stock solutions were separately prepared by dissolving in ethanol nearly 25.0 $( \pm 0.0001 \mathrm{~g}) \mathrm{mg}$ of each drug in $100 \mathrm{~mL}$ volumetric flasks. A first set of 9 binary calibration samples was built by combining five levels of PAR and DPH within the range 1.0-12.0 $\mathrm{mg} / \mathrm{L}$.A second calibration set of nine ternary mixture solutions was prepared for PAR-CAF-PHE and PAR-PSE-CHL systems. For PARCAF-PHE, drug ranges were 2.0-15.0 mg/L for PAR, 2.0-16.0 mg/L for CAF and 1.0-8.omg/L for PHE. For PAR-PSE-CHL, drug ranges were1.0-16.0 mg/L for PAR, 1.0-8.0 mg/L for PSE, and 1.0-12.0 mg/L for CHL. Calibration set of the binary mixtures was created by following a full experimental design while calibration sets of binary systems selected according to Brereton `s rule using three different concentration levels. To validate the PLS and CAP-PLS models, three independent external validation sets consisting of 7 solutions for binary and ternary systems were prepared. Marketed formulations were assayed by weighing four tablets for binary system and five tablets for ternary systems and grinding the tablets to a fine powder. The powder was suspended in water and diluted to a final volume of $100 \mathrm{ml}$. The suspension was sonicated for 10 min, filtered through a $0.45 \mu \mathrm{m}$ filter, and properly diluted in preparation for spectral analysis.

\section{Partial least squares and net-analyte signal calculations}

A double beam UV-visible spectrophotometer (Thermo scientific. Genesys $10 S$ UV-VIS (USA)) with quartz cuvette cell of $1.0 \mathrm{~cm}$ path length was employed. The spectral bandwidth was $1.0 \mathrm{~nm}$ with fast wavelength-scanning speed. Scans were carried out in the range of 200-300 $\mathrm{nm}$ at (1.0 $\mathrm{nm}$ step, 101 points/ spectrum). The spectral data obtained for calibration mixtures are placed in matrix A (size 101×9) and spectral data of validation set are placed matrix $\mathbf{B}$ (size 101×7). On the other hand, concentration data are placed matrix $\mathbf{C}$ (size $9 \times 3$ for ternary systems and size $9 \times 2$ for binary system). Partial least squares PLS1 is an efficient tool for developing a quantitative relationship between several predictor variables $\mathbf{A}$ (spectral data) and a property of interest $\mathbf{c}$ (the independent variable or drugs content) as shown below ${ }^{11,15}$ :

$\mathbf{c}=\mathbf{A b}$

Where c contains the concentrations of calibrated drug in calibration samples arranged in $\mathbf{a}$ vector and $\mathbf{b}$ is the calibration sensitivity which is necessary for estimating drugs content in the adsorption solutions ${ }^{11,16-18}$ :

$\mathbf{b}=\mathbf{W}^{\mathrm{t}}\left(\mathbf{P} \mathbf{W}^{\mathrm{t}}\right)^{-1} \mathbf{q}$ 
Where $\mathbf{W}$ is the weights matrix for $\mathbf{A}, \mathbf{P}$ is the loadings matrix of $\mathbf{A}, \mathbf{q}$ is the loadings vector for $\mathbf{c}, \mathbf{t}$ donates transpose operation, and $\mathbf{- 1}$ stands for inverse operation. Once $\mathbf{b}$ is estimated, prediction of drug concentrations $\left(\mathbf{c}_{\mathrm{un}}\right)$ from the unknown spectrum $\mathbf{a}$ is carried out as following ${ }^{11,16-18}$ :

$\mathbf{c}_{\mathrm{un}}=\mathbf{a b}$

Optimum number of PLS-latent variables were estimated using cross-validation method $^{15}$. The predicted concentrations were compared with the known concentrations of the compounds in each calibration sample. The prediction error sum of square (PRESS) and the relative error of prediction (REP \%) were calculated for drugs in calibration and validation samples as following ${ }^{17,19}$ :

$$
\text { PRESS }=\sum_{i=1}^{n}\left(C_{i, \text { actual }}-C_{i, \text { pred }}\right)^{2}
$$

$\% \operatorname{REP}=100 \times\left(\frac{\sum_{i=1}^{n}\left(C_{i, \text { pred }}-C_{i, a c t}\right)^{2}}{\sum_{i=1}^{m}\left(C_{i, a c t}\right)^{2}}\right)^{1 / 2}$

Where $C_{\text {actual }}, C_{p r d}$, and $n$, are actual concentration (mg/L), predicted concentration obtained by PLS (mg/L), and number of training samples (solutions), respectively. A unifying framework for calibration and prediction in multivariate calibration is shown based on the concept of the Net Analyte Signal (NAS). NAS is the part of the measured spectrum that the calculated model uses for prediction. The main equation that is needed to estimated figures of merit is ${ }^{15,20,21}$ :

$S_{\mathrm{k}}^{*}=\left[\mathrm{I}-\mathrm{S}_{-k} \mathrm{~S}_{-k}^{+}\right] \mathrm{s}_{k}$

Where Sis the matrix of sensitivities collected for all other solutes, $\mathrm{s}_{k}$ is the sensitivity vector of the analyte, and $\mathrm{s}_{\mathrm{k}}{ }^{*}$ is the spectrum of pure analytek measured at unite concentration (sensitivity factor). Sensitivity (SEN) value of analytek is the norm ( \|\|$)$ of the net sensitivity vector, it was defined as the amount of net signal that, in prediction, corresponds to a concentration equal to unity ${ }^{21}$ :

$\mathrm{SEN}=\left\|\mathrm{S}_{k}^{*}\right\|$

Selectivity (SEL) can be defined as the part of the measured signal unique to the analyte of interest. Based on NAS theory, the selectivity measures the extent of spectral overlapping. SEL can be expressed by the ratio between the norm of the NAS vector and the norm of the spectra. SEL values are extended 
from zero (high overlap with other interferences) to unity (no overlap with other sample components). High values of SEN are an indication of high method accuracy for that analyte ${ }^{15,20,21}$ :

$\mathrm{SEL}=\left\|\mathrm{s}_{k}^{*}\right\| /\left\|\mathrm{s}_{k}\right\|$

The limit of detection (LOD) obtained assumes that the prediction uncertainties are approximately constant. LOD gives the minimum detectable amount of $k$ solute. A reasonable estimation to determine the LOD can be estimated $\mathrm{as}^{21}$ :

$\mathrm{LOD}=3\|\varepsilon\| /\left\|\mathrm{s}_{k}{ }^{*}\right\|$

Where $\|\varepsilon\|$ is a measurement of the instrumental noise, and $\mathrm{s}^{*}$ was defined above. Then the norms of blank readings $\left(\left\|\mathrm{NAS}_{\text {blank }}\right\|\right)$ are estimated and $\|\varepsilon\|$ is taken as the standard deviation of estimated norms ${ }^{21}$.The limit of quantification (LOQ) is the minimum quantifiable amount of the solute, is estimated $\operatorname{as}^{15,21}$ :

$\mathrm{LOQ}=10\|\varepsilon\| /\left\|\mathrm{s}_{k}^{*}\right\|$

All calculations were carried out using special matlab-codes under MATLAB ${ }^{\circledR}$.

\section{Cumulative area pre-processing of spectral data prior to PLS calibration}

Data treatment by CAP transforms the original UV spectrum to a new curve using the area underlying the same spectrum. This elaboration is based on two mathematical steps. In the first step, the area $a_{\lambda i}$ binning of the two consecutive wavelengths $\left(\lambda_{i}\right.$ and $\left.\lambda_{i+1}\right)$ is calculated as ${ }^{7}$ :

$$
a_{\lambda i}=\frac{a b_{\lambda i}+a b_{\lambda i+1}}{2}
$$

Where $a b$ is the absorbance value at a single wavelength. This calculation is repeated for all the wavelengths in the spectrum. In the second step, the cumulative sum of the single areas is computed, in such a way as the cumulative area at each wavelength $A_{\lambda i}$ resulted from the sums of all areas $a_{i}$ of the preceding wavelengths. For a spectral region between $\lambda_{m}$ and $\lambda_{n}\left(\lambda_{m}<\lambda_{n}\right)$, the cumulative area $A_{\lambda m, n}$ is estimated as?:

$$
A_{\lambda m, n}=\sum_{i=\lambda m}^{\lambda n} a_{\lambda i}
$$


Treatment of the original UV spectra using CAP (Eqs 11 and 12) offers many advantages including amplification of the analytical signals for all solutes and possible quantification

\section{RESULTS AND DISCUSSION}

\section{Influence of solution $\mathrm{pH}$ on spectral behavior of pharmaceuticals}

Influence of solution $\mathrm{pH}$ on spectral behavior was studied for all drugs at $\mathrm{pH}$ 3.0, 7.0, and 10.0 to cover all possible conditions. Among the drugs, identical spectral shapes over the range (230-280 nm) were observed for CHL and PSE and hence there was no need to present their profiles. On the other hand, the UV-absorption behavior of PAR (which present in all systems), CAF, PHE, and DPH were notably changed with solution $\mathrm{pH}$ as depicted in Fig 1.
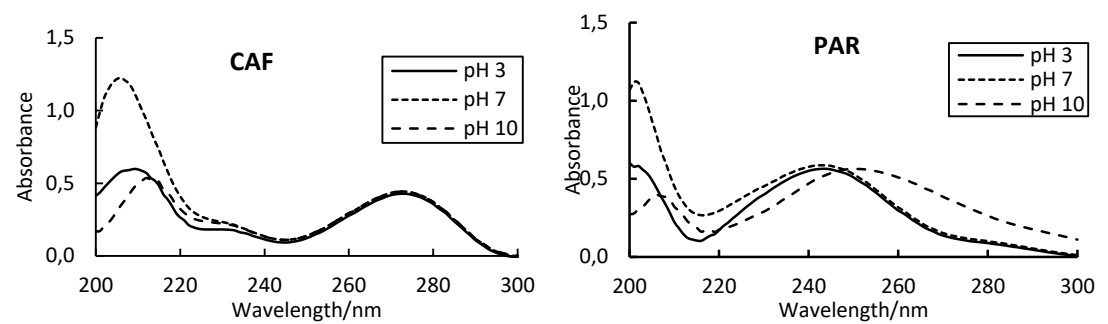

DPH
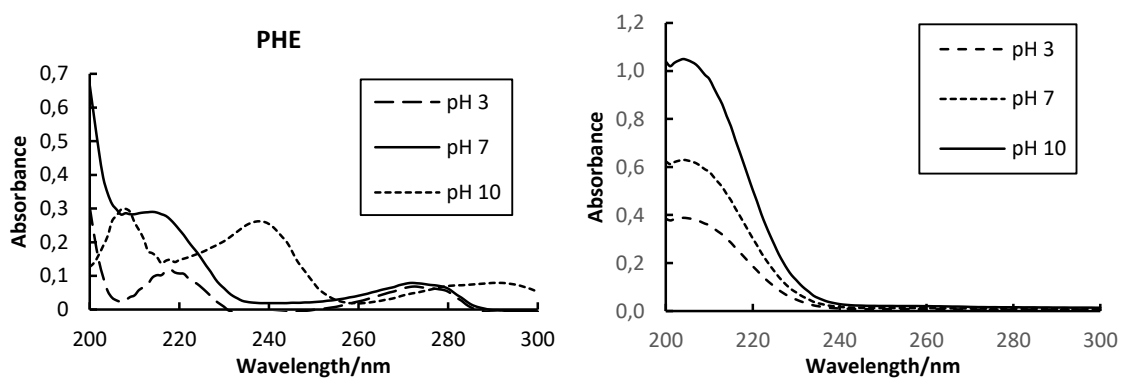

Figure 1. Influence of $\mathrm{pH}$ on spectral behavior of tested drugs $(7.0 \mathrm{mg} / \mathrm{Leach} \mathrm{drug})$

As shown in Fig 1, large variation was noted in the spectra of CAF at the studied $\mathrm{pH}$ and over the range (200-240 $\mathrm{nm}$ ) and slight variations were observed over the rest of the domain. In the same time, the distinct wavelength positioned at 273 $\mathrm{nm}$ was not affected with solution $\mathrm{pH}$. More variations were observed in the PAR spectra, the intensity was notably increased at $\mathrm{pH}$ over the range $200-220 \mathrm{~nm}$ while the main peak (243 nm) was shifted to $250 \mathrm{~nm}$ at $\mathrm{pH}$ 10.0. The most drastic 
changes were reported for PHE as shown in Fig 1. It was highly possible that PHE underwent chemical reaction at the basic medium and this was deduced from the developed spectrum which has two wavelengths at 208 and $237 \mathrm{~nm}$. To avoid PHE side-reaction, it was practical to run UV measurements at $\mathrm{pH}$ 7.0. As can be noted from the earlier discussion, the optimum $\mathrm{pH}$ for spectral analysis is 7.0 which maintain stable structure and high UV absorption for all drugs. For DPH, there was a significant light absorption with $\mathrm{pH}$ over the range $(200-230 \mathrm{~nm})$, while, $\mathrm{pH}$ has no effect on the spectrum over the rest of the domain. $\mathrm{pH} 7.0$ seems to be a good choice to run spectral measurement for this drug. In fact, protonation, hydrolysis, and internal-arrangement of drug molecules are highly possible at acidic or basic solutions and this affects their spectral properties. Accordingly, solution $\mathrm{pH}$ should be adjusted to get unique spectral characteristics of the measured drug.

\section{Spectral behavior of pharmaceuticals and spectral overlapping}

In fact, intense spectral overlapping among pharmaceuticals would retard their quantification in solution and in real formulation ${ }^{11,15}$. Accordingly, the spectral behavior of pharmaceuticals and extent of overlapping were evaluated before running multivariate calibration. The UV spectra of the studied systems are presented in Fig. 2
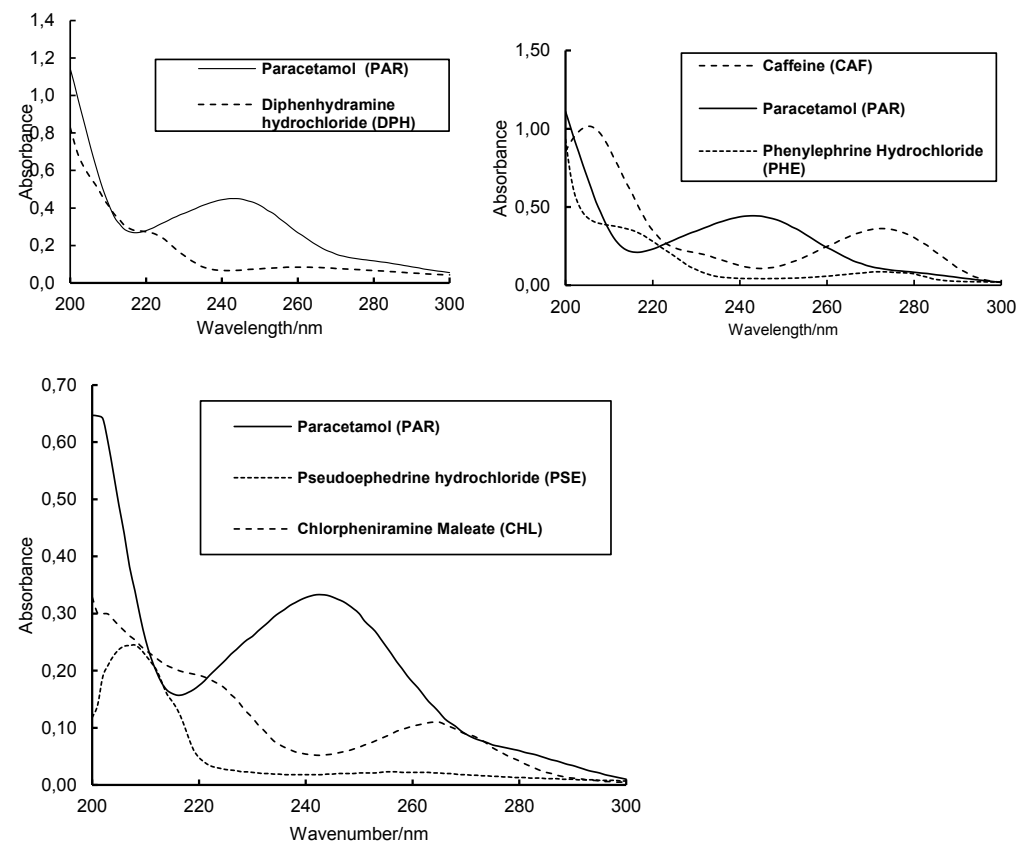

Figure 2. UV Spectra of the binary and ternary drug systems. Top PAR-DPH $(6.0 \mathrm{mg} / \mathrm{L}$ and pH 7.0), Middle CAF-PAR-PHE (7.0 mg/L and pH 7.0) Bottom PAR-PSE-CHL (5.0 mg/L and $\mathrm{pH} 7.0)$ 
As indicated in Fig. 2, all drugs exhibited strong light absorption over the range $(200300 \mathrm{~nm})$ in addition to intense overlapping among the signals. For PAR$\mathrm{DPH}$, a large spectral overlap was observed between both compounds and particularly over the range $220-280 \mathrm{~nm}$. In addition to intense overlapping, PAR has better UV absorption compared to DPH and would make their quantification by simple spectrometry (especially DPH) a hard task. The intense absorption of PAR at $243 \mathrm{~nm}$ was mainly attributed to the $n-\pi^{*}$. One more point on this PAR-DPH is the presence of DPH in much lower amount compared to PAR in real formulation and this added more analytical obstacles on their accurate quantification. For the ternary system CAF-PAR-PHE, the solutes were actively absorbing over the region this was attributed to their chemical structure which contains many active functional groups. The spectra of the drugs indicated the following distinct absorption wavelengths at 243, 273, and (215 and $272 \mathrm{~nm}$ ) for PAR, CAF and PHE, respectively. The reported absorption wavelengths were mainly attributed to $n \rightarrow \pi^{*}$ and $\pi \rightarrow \pi^{*}$ electronic transitions in the molecules. In fact, the longer maximum wavelength of absorption observed for CAF $(273 \mathrm{~nm})$ was attributed to the conjugated system as indicated from the chemical structures of the drugs (Table 1). Generally, PHE showed weaker absorption compared to other drugs and absorb near the lower end of the spectral range $(220 \mathrm{~nm})$. The spectral overlap between drugs is high over the entire spectral range. Within the spectral regions 250-270, an intense overlapping between PAR and CAF was observed and this would affect their quantification in real formulations. Moreover, PHE absorbs over the entire range. In this ternary system, PHE should be added in much lower levels and this negatively reflected on its quantification in real formulations. For PAR-PSE-CHL, the solutes were active in UV region and this is attributed to their chemical structure which contains aromatic parts substituted with functional groups (Table 1). The spectra of drugs indicated the following special absorption wavelengths 210, 242, and $265 \mathrm{~nm}$ for PSE, PAR and CHL, respectively. The observed bands were mainly attributed to $n \rightarrow \pi^{*}$ and $\pi \rightarrow \pi^{*}$ electronic transitions. Generally, PSE has a poor UV absorption compared to two drugs and absorb near the extreme of the applied spectral domain. In fact, the spectral overlap between drugs is high over the entire spectral range. Within the spectral regions 220-240 and 260-280 $\mathrm{nm}$, an intense overlapping between PAR and CHL was observed (Fig. 1). Moreover, PSE absorbs over the entire range. Practically, PAR and PSE are added in much higher levels compared to $\mathrm{CHL}$ and this would make quantification of the later drug a hard-analytical task. For each system, the extent of spectral overlapping between drugs was estimated using net-analyte signal calculations (Eq. 8) and the results are provided in Table 2. 
Table 2. Extent of spectral overlapping in the drug systems using NAS calculations ${ }^{\mathrm{a}}$

\begin{tabular}{|c|c|}
\hline \multicolumn{2}{|c|}{ Total spectral overlapping with other drugs $\%$} \\
\hline PAR-PSE-CHL \\
\hline PSE & 53 \\
\hline CHL & 55 \\
\hline \multicolumn{2}{|c|}{ CAF-PAR-PHE } \\
\hline CAF & 68 \\
\hline PAR & 85 \\
\hline PHE & 79 \\
\hline PAR & 38 \\
\hline DPH & 62 \\
\hline
\end{tabular}

aFor PAR-PSE-CHL, NAS calculations were carried out at $5.0 \mathrm{mg} / \mathrm{L}, \mathrm{pH} 7.0$, and spectral range $200-300 \mathrm{~nm}$. For CAF-PAR-PHE, calculations were carried out at $7.0 \mathrm{mg} / \mathrm{L}, \mathrm{pH} 7.0$ and 200-300 nm. For PAR-DPH, 6.0 mg/L, pH 7.0 and 200-300 nm.

As indicated in Table 2, a significant overlapping among drugs was reported and the intense overlapping was in the ternary system (CAF-PAR-PHE) which extended from $68 \%$ to $85 \%$. For the earlier ternary system, PAR exhibited an intense overlapping with $\mathrm{CAF}$ and $\mathrm{PHE}$ which may negatively reflect on its accurate quantification in solution or in real extracts. In fact, PAR is added many-folds higher than other two drugs in the formulation which may not affect its final measurements. On the other hand, the high overlapping of CAF and PHE (68\%-79\%) is negatively affect their final quantification taking into account their lower levels compared to CAF in real formulations. This ternary system represented a real challenge for multivariate calibration methods due to the intense spectral overlapping. The same discussion is holding true for other systems. The most problematic issue in the ternary system CAFPAR-PHE is the intense overlapping of PHE with other drugs (79\%) while being present in very little amount compared to the rest of drugs. The same is true for CAF as it added in modest amounts compared to PAR which added in much higher dosages. In the same manner, accurate quantification of DPH (spectral overlapping 62\% with PAR) in real formulations may not be a straightforward analytical job. In addition to spectral overlapping, the presence of un-calibrated excipients in the extra will add more interference on analysis and hence reduce the performance of multivariate calibration methods ${ }^{11,16,17}$. 
Pre-processing of the UV spectral data by CAP: Signal amplification

For better assessment on signal prepossessing before PLS calibration, UV spectra of all drugs and their mixtures (binary and ternary) were recorded at the ratios identical to the real formulations along with CAP spectral transformation. The results are shown in Fig 3.
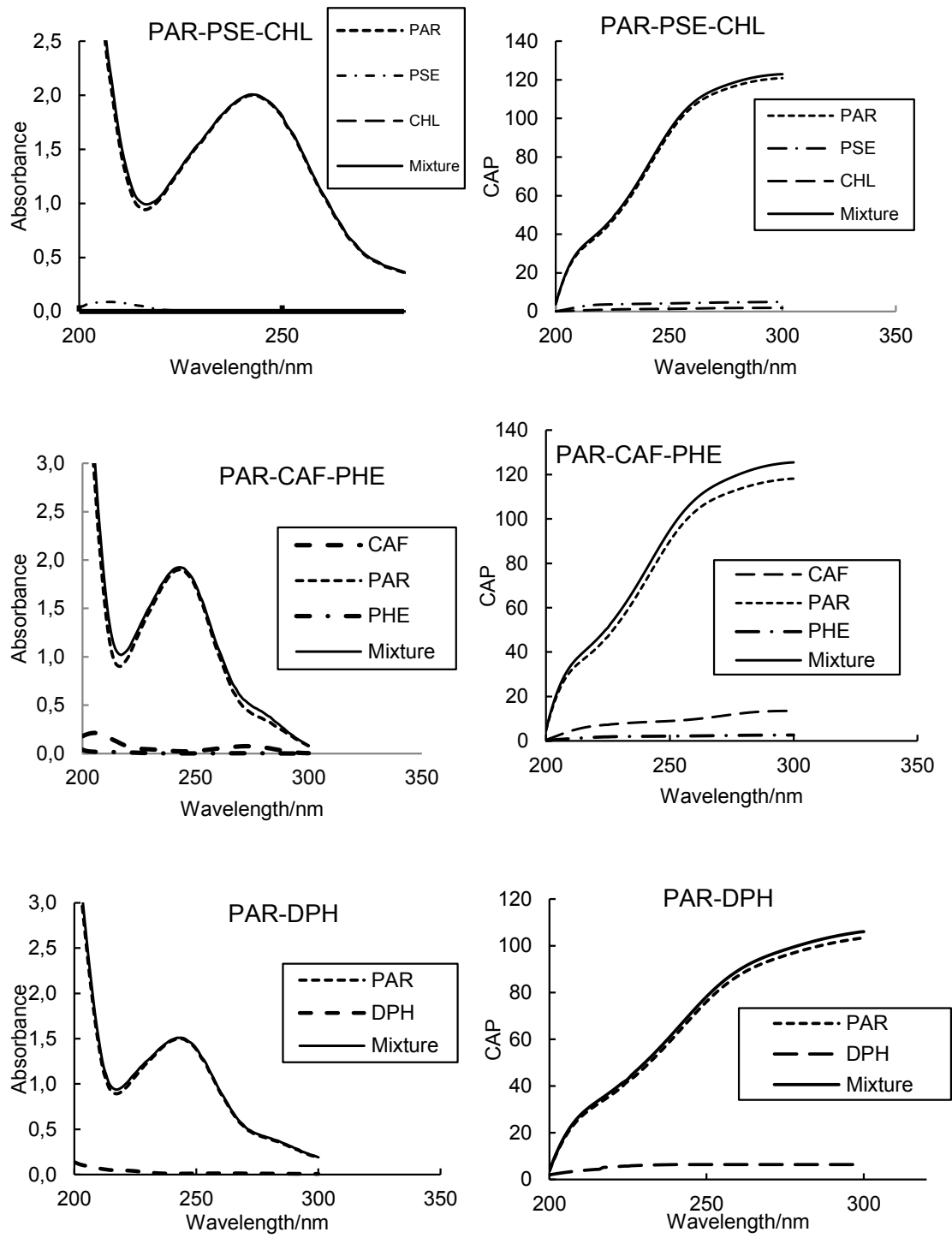

Figure 3. UV spectra and CAP transformation of drugs and their mixtures recorded the same ratios as in real formulations. 
In the three systems, UV spectra indicated a strong absorption signal of PAR due its higher concentration. For PAR-PSE-CHL, PAR was added in excess than CHL and CHL by 150 and 10 times, respectively. While for PAR-CAFPHE, PAR was 100 and 20 times higher than PHE and CAF, respectively. For the binary system, PAR was 20-times higher than the other drug. The high level of PAR in the systems would explain the similarity between PAR and the spectrum of the mixture in all cases. The difficulty in analyzing the mixtures was increased by the fact that the absorptivity values of CAF, CHL, PSE and DPH in the respective formulations were much lower than the other PAR over the entire spectral domain, making their accurate determination a hard-analytical task unless the components are separated before detection. This difficulty drastically reduced the chance to apply classical spectroscopic methods which often adopted discrete wavelengths for detection. In these cases, multivariate calibration methods seem more suitable because they use simultaneously a large number of signals per spectrum. However, considering the difficulty in solving this type of mixtures, an appropriate pre-processing of the analytical data seemed necessary in order to minimize any instrumental interference and at the same time select the most useful information to emphasize the contribution of substances of low concentration.

As outlined earlier, CAP treatment transforms the original UV spectrum into a new curve created by using the area underlying the spectrum. This treatment is based on two mathematical steps as outlined earlier. Indeed, the conversion of original UV spectra through the CAP method provided many advantages among which the amplification of the analytical signals. Fig 3 shows the outputs of CAP treatment of the spectral of the three systems where drugs recorded at their actual ratios. The major differences in comparing the original absorbance curves with CAP plots are: a) the magnitudes on the $y$-axis indicated a high amplification of the signal and this supposed to increase the sensitivity of the quantitative measurements and help in quantifying the components even at trace levels (like PSE, CHL, CAF and DPH in the presence of PAR), b) CAP was able to magnify the weak signals of PSE, CHL, CAF and DPH and over the whole spectrum and this option is rather fundamental when applying multivariate techniques which utilize the full spectrum to extract the maximum analytical information, and c) Unlike UV signal, the values of CAP signal were stabilize at constant value and this was reported for all drugs. This stable value, in fact, is due to the additive contribution of all intensities at all wavelengths. It was reported that CAP filtering would end up with accurate quantification of drugs when present at trace levels ${ }^{7}$. The analytical matrices were built on the spectral data. In the matrix $\mathbf{A}$ (dependent variables) each sample was described by $n$ 
variables, corresponding to the wavelengths and to the respective absorbance values; in the matrix $\mathbf{C}$ (independent variables) the samples were described by the concentrations of each component. Two data matrices for each drugsystem were created; the first matrix contained the untreated spectral data while the other matrix contained the spectral data upon CAP. The calibration curves relative to the binary (PAR-DPH) and ternary systems (PAR-PSE-CHL and PAR-CAF-PHE) along with CAP transformation are presented in Figure 4.
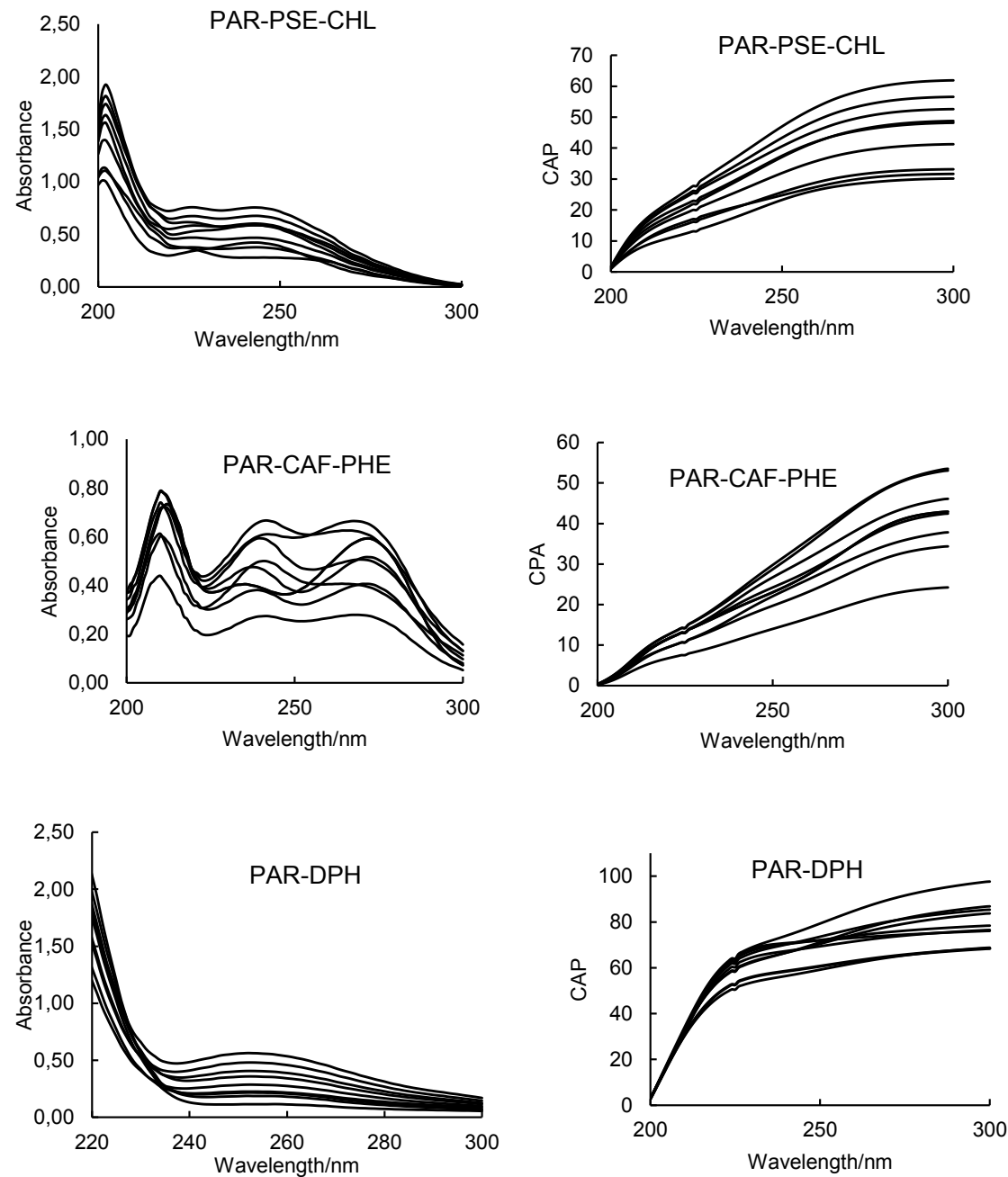

Figure 4. UV spectra of PAR-DPH, CAF-PAR-PHE and PAR-PSE-CHL calibration samples and plots after CAP transformation. 


\section{Determination of pharmaceuticals in binary and ternary}

drugs systems by PLS

PLS and CAP-PLS can be created and applied to predict drugs without previous knowledge on calibrated solutes. However, intense overlapping between drugs and strong influence of excipients would affect the prediction power of $\mathrm{PLS}^{15,16}$. The performance of before and after signal filtering drugs prediction in validation set is provided in Table 3 .

Table 3. External prediction of drugs in different systems.

\begin{tabular}{|c|c|c|c|c|c|c|}
\hline \multirow{2}{*}{ Drug } & \multicolumn{5}{|c|}{ PLS $^{\mathbf{2}}$} & \multicolumn{3}{c|}{ CAP-PLS $^{\mathbf{2}}$} \\
\cline { 2 - 7 } & PRESS & REP\% & Rec\% & PRESS & REP\% & Rec\% \\
\hline \multicolumn{7}{|c|}{ PAR-PSE-CHL } \\
\hline PAR & 0.32 & 3.9 & $96.3(4)$ & 0.06 & 1.7 & $98.0(3)$ \\
\hline PSE & 2.95 & 12.6 & $95.2(5)$ & 0.13 & 3.1 & $97.7(4)$ \\
\hline CHL & 2.32 & 11.2 & $94.0(5)$ & 0.15 & 2.5 & $97.7(4)$ \\
\hline \multicolumn{7}{|c|}{ PAR-CAF-PHE } \\
\hline PAR & 3.41 & 14.0 & $106.8(5)$ & 0.82 & 5.8 & $99.2(3)$ \\
\hline CAF & 2.93 & 10.0 & $97.4(4)$ & 0.45 & 4.3 & $103.6(3)$ \\
\hline PHE & 1.54 & 8.0 & $95.8(4)$ & 0.18 & 2.1 & $101.4(3)$ \\
\hline \multicolumn{7}{|c|}{ PAR-DPH } \\
\hline PAR & 0.45 & 3.4 & $96.9(3)$ & 0.14 & 2.1 & $98.5(2)$ \\
\hline DPH & 0.64 & 4.9 & $95.7(5)$ & 0.19 & 3.0 & $97.0(4)$ \\
\hline
\end{tabular}

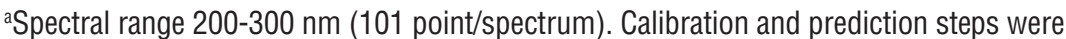
carried out using Eqs1-3. Calibration and validation sets are provided in Table 1. PLS variables were provided between brackets for each system.

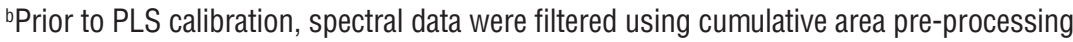
method (Eqs 11\&12) and PLS variables were provided between brackets for each system.

As shown in Table 3, PLS was workable to predict PAR-PSE-CHL and the best prediction observed for PAR with a REP value 3.9\%. In general, PLS was not so-effective for predicting PSE in the mixtures with a high REP $12.6 \%$ which may not acceptable for pharmaceutical analysis. The modest prediction of PSE was mainly attributed to the intense spectral-overlapping with other drugs (Table 2). Accordingly, application of PLS for PSE and CHL may not be workable taking into account the negative influence of excipients in real cases. As indicated in Table 3, the performance of CAP-PLS was very promising with 
REP values of $1.7 \%, 2.5 \%$, and 3.1\% for PAR, CHL, and PSE, respectively, and this reflected the high closeness between nominal and predicted values. The earlier results showed that CAP-PLS outperformed PLS for predicting PSE and CHL in ternary systems. In general, CAP-PLS was excellent for predicting all drugs with PRESS values less than 1.0 in all cases. The same results were noted for PAR-CAF-PHE where CAP-PLS outperformed PLS for drugs quantification with final REP 2.1-5.8\%. The best prediction was reported for PHE down to $1.0 \mathrm{mg} / \mathrm{L}$ in mixtures rich with PAR and CAF. Due to the lesser spectral overlapping, both PLA and CAP-PLS were both of comparable performance for PAR-DPH quantification, REP was all lower than 5\% for both methods. The superiority of CAP-PLS was mainly attribute to the effective signal filtering prior to PLS calibration which improved final prediction? ${ }^{7}$

\section{Quantification of drugs in real formulations}

The real testing of the proposed CAP-PLS method will be assessed by analyzing binary and ternary systems in the commercial formulations. Direct analysis of drugs in commercial tablets is not an easy task due to the presence of drugs in unequal quantities and also the presence of excipients that did not involve while constructing calibration mixtures. In addition to the above, the drugs are often placed at concentrations far higher than the calibration solutions. Therefore, all the ingredients should be properly diluted to be measured with acceptable sensitivity. In this case, signal filtering/enhancing will be essential before running classical PLS calibration. It is rather essential to mention that matrix-cleaning methods may be adopted to eliminate/reduce the influence of interferences before running analysis ${ }^{22-24}$. It was necessary to evaluate the effect of dilutions on drugs quantification. The spectral shapes of the systems and at variable dilutions are provided in Figure 5. Drugs quantification in real formulations is provided in Table 4 and the PLS outputs are provided for comparison purposes. 

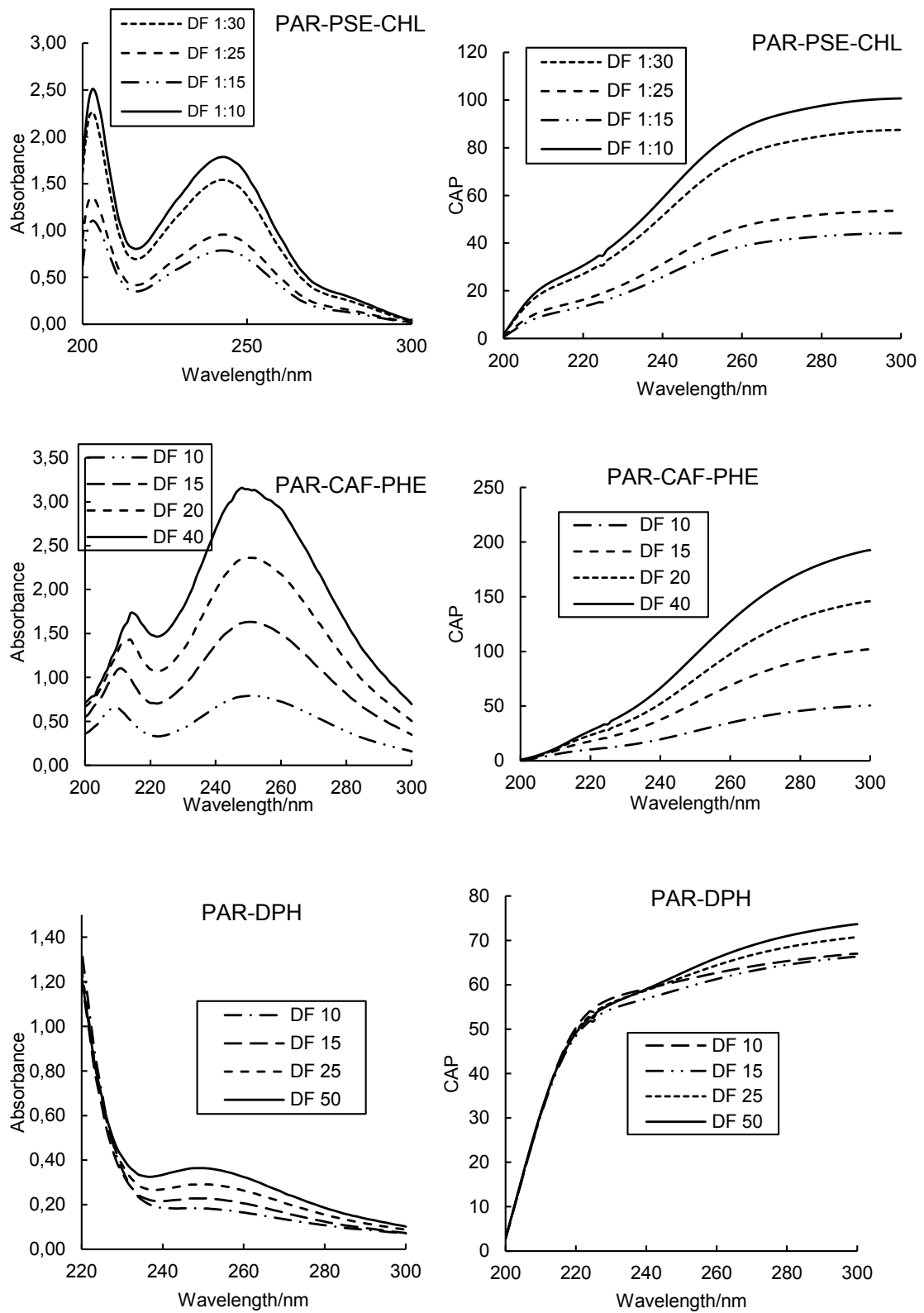

Figure 5. UV spectra of marketed formulations after extraction and dilution with distilled water and CAP plots. 
Table 4. Implementation of PLS and CAP-PLS for drugs quantification on different systems.

\begin{tabular}{|c|c|c|c|c|c|c|c|}
\hline \multirow[b]{2}{*}{ System } & \multirow[b]{2}{*}{$\begin{array}{c}\text { Claimed } \\
\text { (mg/tablet) }\end{array}$} & \multicolumn{3}{|c|}{ PLS } & \multicolumn{3}{|c|}{ CAP-PLS } \\
\hline & & $\begin{array}{l}\text { Predicted } \\
\text { (mg/tablet) }^{\mathrm{a}}\end{array}$ & $\begin{array}{c}\text { Mean } \\
\text { Recovery } \\
\%(n=3)\end{array}$ & $\begin{array}{c}\text { Precision } \\
(n=3, \text { RSD } \%)\end{array}$ & $\begin{array}{l}\text { Predicted } \\
\text { (mg/tablet) }\end{array}$ & $\begin{array}{c}\text { Mean } \\
\text { Recovery } \\
\%(n=3)\end{array}$ & $\begin{array}{c}\text { Precision } \\
(n=3, \text { RSD \%) }\end{array}$ \\
\hline \multicolumn{8}{|l|}{ PAR-CAF-PHE } \\
\hline PAR & 500 & 503.0 & 100.6 & 8.4 & 501.0 & 102.6 & 2.1 \\
\hline CAF & 25 & 26.0 & 104.0 & 6.5 & 24.7 & 103.0 & 3.1 \\
\hline PHE & 5 & 4.5 & 90.0 & 7.3 & 5.1 & 94.3 & 4.2 \\
\hline \multicolumn{8}{|c|}{ PAR-PSE-CHL } \\
\hline PAR & 300 & 508.0 & 99.0 & 5.0 & 504.0 & 98.9 & 3.1 \\
\hline PSE & 30 & 28.7 & 102.0 & 7.0 & 25.2 & 104.6 & 2.7 \\
\hline $\mathrm{CHL}$ & 2 & 1.8 & 99.0 & 4.6 & 2.1 & 99.0 & 1.5 \\
\hline \multicolumn{8}{|l|}{ PAR-DPH } \\
\hline PAR & 500 & 503 & 101.2 & 2.1 & 502 & 104.2 & 0.8 \\
\hline DPH & 25 & 28 & 105.3 & 3.1 & 24 & 106.3 & 1.0 \\
\hline
\end{tabular}

As indicated in Fig 5, identical UV spectra and CAP signals were observed for the systems. Higher intensities (for CAP signals) were observed at lower dilutions (DF 10 and 15). Initial analysis indicated that running PLS or CAP-PLS using signals recorded at DF 10 or 15 ended up with high prediction errors. In fact, at lower DF the content of drugs is much higher than those used in calibration mixtures making external prediction is not valid. As already known, the external prediction of solutes is often dependent on the ranges selected in the calibration mixtures. In the current systems, the best prediction in real formulations was achieved at DF 15, 20, and 25 for PAR-DPH, PAR-CAF-PHE, and PAR-PSE-CHL, respectively. Both models were used to assay drugs in real formulations which based the same composition of the calibration mixtures. The results, provided in Table 4, indicated the high performance of CAP-PLS for accurate quantification of drugs in binary and ternary systems. For ternary mixtures, CAP-PLS manifested better accuracy than PLS with recoveries 94.3\%-102.6\% and precisions (RSD) 2.1-3.1\% for PAR-CAF-PHE and 98.9\%104.6\% and 1.5-3.1 for PAR-PSE-CHL. It seems that signal filtering by CAP has passively reflected on the final PLS performance. For the other binary system, a good agreement was observed between experimental and nominal levels (obtained by both models) of the commercial formulation. Again, PLS calibration created using CAP-filtered-data generated the best results and may properly handed the negative influence of excipients present in the dosage forms. 
UV-signal filtering by CAP prior to PLS was found more effective than PLS when handling ternary drug mixtures. The proposed CAP-PLS can handle systems in which one component present at lower levels in the formulation. CAP simply estimated the cumulative sum of the areas under curve between two consecutive wavelengths which improved the analytical signal. The proposed CAP-PLS was effective to predict two ternary systems (PAR-CAF-PHE and PAR-PSE-CHL) of intense overlapping and containing one ingredient available at lower concentration. For binary system, application of CAP-PLS was as good as classical PLS along with raw UV signals. CAP, as an appropriate pretreatment method, have been succeeded for accurate and simultaneous quantification of commercial pharmaceutical formulations in which the ratio between the components is highly variable.

\section{ACKNOWLEDGEMENTS}

The authors thank Deanship of Graduate Studies (The Hashemite University, Jordan) and for the financial support to this research. The researchers also thank all the technical staffs at chemistry department for their continued help.

$\begin{array}{ll}\text { List of abbreviations } \\ \text { CAF } & \text { Caffeine } \\ \text { CHL } & \text { Chlorpheniramine Maleate } \\ \text { CAP } & \text { Cumulative Area Pre-Processing } \\ \text { DPH } & \text { Diphenhydramine Hydrochloride } \\ \text { LOD } & \text { Limit of Detection } \\ \text { LOQ } & \text { Limit of Quantification } \\ \text { NAS } & \text { Net Analyte Signal } \\ \text { PLS } & \text { Partial Least Squares } \\ \text { PRESS } & \text { Prediction Error Sum of Square } \\ \text { PSE } & \text { Pseudoephedrine Hydrochloride } \\ \text { REP\% } & \text { Relative Error of Prediction } \\ \text { SEL } & \text { Selectivity } \\ \text { SEN } & \text { Sensitivity }\end{array}$




\section{REFERENCES}

1. Shaikh, K.; Devkhile, A. Simultaneous determination of aceclofenac, paracetamol, and chlorzoxazone by RP-HPLC in pharmaceutical dosage form. J. Chroma. Sci. 20o8, 46, 649652.

2. Ali, N.; Zaazaa, H.; Abedelkawy, M.; Magdy, M. Simultaneous determination of paracetamol and diphenhydramine hydrochloride in presence of paracetamol degradation product. $J$. Pharm. Anal. Acta. 2011, 2, 9.

3. Dou, Y.; Sun, Y.; Ren, Y.; Ren, Y. Artificial neural network for simultaneous determination of two components of compound paracetamol and diphenhydramine hydrochloride powder on NIR spectroscopy. Anal. Chim. Acta. 2005, 528, 55-61.

4. Goicoechea, H.; Olivieri, A. Simultaneous multivariate spectrophotometric analysis of paracetamol and minor components (diphenhydramine phenylpropanolamine) in tablet preparations. J. Pharm. Biomed. Anal. 1999, 20. 255- 261.

5. Hegazy, M.; Abbas, S.; Zaazaa, H. Resolution of overlapped quaternary spectral bands by net analyte signal based methods; an application to different combinations in tablets and capsules. J. Anal. Chem. 2015, 70, 450-458.

6. De Luca, M.; Ioele, G.; Spatari, C.; Ragno, G. Optimization of wavelength range and data interval in chemometric analysis of complex pharmaceutical mixtures. J. Pharm. Anal. 2016, 6, 64-69.

7. De Luca, M.; Ioele, G.; Ragno, G. Cumulative area pre-processing (CAP): A new treatment of UV data for the analysis of complex pharmaceutical mixtures. J. Pharm. Biomed. Anal. 2014, $90,45^{-} 51$.

8. Singh, V.; Daharwal, S.; Suresh, P. A review of instrumental analytical methods to assay active ingredients in multicomponent pharmaceutical formulations. Columb. J. Pharm. Sci. 2014, 1, 27-39.

9. Qi, M.; Wang, P.; Zhou, L.; Sun, Y. Simultaneous determination of four active components in a compound formulation by liquid chromatography. Chromatographia. 2003, 58, 183186.

10. Belal, T.; Abdel-Hay, K.; Clark, R. Selective determination of dimenhydrinate in presence of six of its related substances and potential impurities using a direct GC/MS method. J. Adv. Res. 2016, 7, 53-58.

11. Gergov, G.; Alin, A.; Doychinova, M.; De Luca, M.; Simeonov, V.; Al-Degs, Y. Assessment of different PLS algorithms for quantification of three spectrally overlapping drugs. Bull. Chem. Comm. 2017, 49, 410-421.

12. Khajehsharifi, H.; Sadeghi, M.; Pourbasheer, E. Spectrophotometric simultaneous determination of ceratine, creatinine, and uric acid in real samples by orthogonal signal correction-partial least squares regression. Chemical Monthly. 2009, 140, 685-691.

13. Gabrielsson, J.; Jonsson, H.; Airiau, C.; Schmidt, B.; Escott, R.; Trygg, J. OPLS methodology for analysis of pre-processing effects on spectroscopic data. Chemom. Intell. Lab. Syst. 2006, 84, 153-158.

14. Svensson, O.; Kourti, T.; MacGregor, J. An investigation of orthogonal signal correction algorithms and their characteristics. J. Chemomet. 2002, 16, 176-188 
15. Al-Degs, Y.; El-Sheik, A.; Issa, A.; Al-Ghouti, M.; Sunjuk, M. Asimple and accurate analytical method for determination of three commercial dyes in different water systems using partial least squares regression. Wat. Sci. Technol. 2012, 66, 1647-1655.

16. Goicoechea, H.; Olivieri, A. Simultaneous determination of phenobarbital and phenytoin in tablet preparations by multivariate spectrophotometric calibration. Talanta. 1998, 47, 103-108

17. Haaland, D.; Thomas, E. Partial least-squares methods for spectral analyses.1. Relation to other quantitative calibration methods and the extraction of qualitative information. Anal. Chem. 1988, 6o, 1193-1202.

18. Andersson, M. A comparison of nine PLS1 algorithms. J. Chemomet. 2009, 23, 518-529

19. Noor, P.; Khanmohammadi, M.; Roozbehani, B.; Garmarudi, A. Evaluation of ATR-FTIR spectrometry in the fingerprint region combined with chemometrics for simultaneous determination of benzene, toluene, and xylenes in complex hydrocarbon mixtures. Chem. Month. 2018, 149, 1341-1347.

20. Lorber, A.; Faber, K.; Kowalski, B. Net analyte signal calculation in multivariate calibration. Anal. Chem. 1997, 69, 1620-1626.

21. Yousefinejad, S.; Hemmateenejad, B. Simultaneous spectrophotometric determination of paracetamol and para-aminophenol in pharmaceutical dosage forms using two novel multivariate standard addition methods based on net analyte signal and rank annihilation factor analysis. Drug test. Anal. 2012, 4, 507-514.

22. El-Sheikh, A; Al-Jafari, M; Sweileh, J. Solid phase extraction and uptake properties of multi-walled carbon nanotubes of different dimensions towards some nitro-phenols and chloro-phenols from water, Int. J. Env. Anal. Chem. 2012, 92, 190-209.

23. El-Sheikh, A; Sweileh, J; Saleh, M. Partially-pyrolyzed olive pomace sorbent of high permeability for preconcentration of metals from environmental waters, J. Hazard. Mat. 2oog, $169,58-64$.

24. Al-Hashimi, N; Aleih, H; Fasfous, I; AlKhatib, H. Multi-Walled Carbon Nanotubes as Efficient Sorbent for the Solid Bar Microextraction of non-Steroidal Anti-Inflammatory Drugs from Human Urine Samples. Curr. Pharm. Anal. 2018, 14, 239-246. 